\title{
ENRAIZAMENTO DE ESTACAS CAULINARES DE KUDZU
}

\section{ROOTING OF STEM CUTTINGS OF KUDZU}

\author{
Michele Fernanda BORTOLINI ${ }^{1}$ \\ Alain DOBIGNIES 2 \\ Katia Christina ZUFFELLATO-RIBAS ${ }^{3}$ \\ Antonio Aparecido CARPANEZZI ${ }^{4}$ \\ Fernando Rodrigues TAVARES ${ }^{4}$
}

\begin{abstract}
RESUMO
Kudzu (Pueraria lobata (Willd) Ohwi) é uma leguminosa semilenhosa, de hábito rasteiro, originária do leste e sudeste asiático, utilizada para alimentação animal; adubação verde; para fins medicinais e culinários; na indústria de papel e tecidos, podendo ainda ser destinada à recuperação de áreas degradadas. Como sua propagação sexuada é dificultada pelo escasso florescimento e pela baixa produção de sementes, a propagação vegetativa pode ser uma alternativa para a produção de mudas dessa espécie. Durante 2003 foram realizados trabalhos na Embrapa Florestas, em Colombo - PR, visando a macropropagação de kudzu, com estacas de $12 \mathrm{~cm}$ de comprimento, $0,7 \mathrm{~cm}$ de diâmetro, sem folhas e com uma gema na porção apical, coletadas nas quatro estações do ano. Como tratamento fitossanitário prévio, as estacas foram imersas em solução de hipoclorito de sódio a $0,5 \%$,

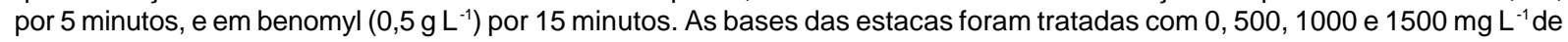
ácido indolbutírico (AIB), depois plantadas em tubetes de polipropileno com vermiculita como substrato e mantidas em casa-devegetação com nebulização intermitente por 30 dias. Foi utilizado o delineamento inteiramente casualizado com 4 tratamentos, 4 repetições de 24 estacas por parcela, totalizando 384 estacas por estação. As melhores porcentagens de enraizamento foram detectadas com $1000 \mathrm{mg} \mathrm{L}^{-1}(61,46 \%)$ e $1500 \mathrm{mg} \mathrm{L}^{-1} \mathrm{AIB}(73,96 \%)$, no verão, e $1500 \mathrm{mg} \mathrm{L}^{-1} \mathrm{AlB}(64,06 \%)$ na primavera. O uso do AIB foi efetivo no enraizamento de estacas de Kudzu, em qualquer estação, exceto inverno.
\end{abstract}

Palavras-chave : estaquia, auxina, AIB, Pueraria lobata

\begin{abstract}
Kudzu (Pueraraia lobata (Willd) Ohwi) is a semihardwood legume of low habitat, from east and southeast of the Asian, used for animal feeding as green fertilizer, for medicinal and culinary ends, to paper and woven manufacturing and it can be destined to the recovery of degraded areas. For pioneering to be considered, with efficiency in the soils covering, As your the sexual propagation of the species is made unfeasible by the scarce flowering and for the low seeds production, the vegetative propagation be can alternative for the production of your seedlings. During 2003 works were accomplished in Embrapa Florestas, in Colombo - PR, seeking the kudzu vegetative propagation, with cuttings made $12 \mathrm{~cm}$ of length, diameter $0,7 \mathrm{~cm}$, without leaves and a yolk in the upper, colleted in the four seasons. As previous sanitary treated the cuttings were immersed in solution of sodium hipiclorite $(0,5 \%)$ for 5 minutes, and in benomyl $\left(0,5 \mathrm{~g} \mathrm{~L}^{-1}\right)$ for 15 minutes. The basis of cuttings were treated with $0,500,1000$ and $1500 \mathrm{mg} \mathrm{L}^{-1}$ of indolebutyric acid (IBA), planted in polypropylene boxes with vermiculite and remaining in a greenhouse under intermittent mist during for 30 days. Data were analyzed using a completely randomized design with 4 treatments, 4 replications of 24 cuttings each, totaling 384 cuttings for season. The best rooting percentages were detected with $1000 \mathrm{mg} \mathrm{L}^{-1}(61,46 \%)$ and $1500 \mathrm{mg} \mathrm{L}^{-1}$ IBA $(73,96$ $\%)$, in summer, and $1500 \mathrm{mg} \mathrm{L}^{-1}$ IBA $(62,06 \%)$ in spring. The use of the IBA it was shown efficient in the rooting of cuttings of Kudzu, any seasons, except in winter.
\end{abstract}

Key-words: cutting, auxin, IBA, Pueraria lobata

\footnotetext{
${ }^{1}$ Bióloga, Doutoranda em Agronomia - Produção Vegetal, Departamento de Fitotecnia e Fitossanitarismo, Universidade Federal do Paraná, UFPR, Curitiba/PR. Bolsista CNPq.

${ }^{2}$ Eng. Agrônomo

${ }^{3}$ Bióloga, Dra., Prof a. Associada, Departamento de Botânica, Setor de Ciências Biológicas, Universidade Federal do Paraná (UFPR). Caixa

Postal 19031, CEP 81531-970, Curitiba, PR. E-mail: kazu@ufpr.br

${ }^{4}$ Eng. Florestal, Dr., Pesquisador Embrapa Florestas, Colombo/PR.
} 


\section{INTRODUÇÃO}

Pueraria lobata (Willd) Owhi, pertencente à família Fabaceae, popularmente conhecida como kudzu, é uma planta perene, semilenhosa de hábito rasteiro ou trepador. Originária do extremo oriente, foi introduzida no Brasil em $1916 \mathrm{com}$ a finalidade de constituir uma cultura forrageira para a alimentação animal e para adubação verde (ROMM,1953; NEME, 1966).

Devido a sua rusticidade, tem grande potencial agronômico e/ou ecológico, pois apresenta tolerância à seca e a solos pobres em nutrientes, além da grande capacidade de adaptação a novos ambientes (DCR, 2003; STARR, et al. 2003 MUNGER, 2003; FORSETH; INNIS, 2004). Como cultura forrageira pode ser explorada em sistemas de rotação, sucessão ou consórcio com culturas anuais e perenes ou na recuperação de áreas, na proteção e conservação dos solos contra a erosão e, na melhoria de suas características físicas, químicas e biológicas (PAEZ, 1946; NEME, 1966; CORRÊIA, 1969).

Além disso, kudzu possui propriedades medicinais, como no tratamento do sarampo, na hipertensão, em enfermidades dos aparelhos óptico e surdez e, mais recentemente tem sido usada no tratamento do alcoolismo (LIU, 2002). Essa planta também pode ser utilizada na culinária, como planta ornamental, no artesanato e para a contenção de laterais em rodovias (DCR, 2003).

Kudzu perde suas folhas e entra em dormência no inverno, voltando a vegetar intensamente na primavera. Seu florescimento é escasso, ocorrendo após 2 anos de estabelecimento da planta na área ocupada. De cada legume produzido, apenas uma ou duas sementes são viáveis (ROCHA, 1964; NEME, 1966; HUGHES, 1978; MUNGER, 2003). Em condições naturais propagase através de sementes previamente escarificadas ou por emissão de raízes das gemas ao longo dos ramos, as quais se difundem sobre o solo ou elevamse sobre outras plantas ou objetos em seu caminho, formando novas plantas a partir destas gemas (KKDS, 2002; APIRS, 2003; STARR, 2003).

A micropropagação desta espécie já foi testada com o intuito de examinar a produção de compostos fenólicos, isoflavonóides, distribuídos por toda planta, e com êxito Thiem (2003), conseguiu com a propagação das plantas, especialmente com o desenvolvimento do sistema radicial uma fonte alternativa de puerarina e daidzeina, compostos farmacologicamente ativos do Kudzu. Na china esses compostos são comumente extraídos das raízes destas plantas (HUA-NENG; CHAO-HONG 2007).

A propagação vegetativa desta espécie também é possível pela técnica de estaquia com ramos jovens ou ainda com coroas de raízes tuberosas (PAEZ, 1946; NEME, 1966; HUGHES, 1978). Como uma alternativa viável, a estaquia possibilita uma multiplicação rápida e prática, sendo este método largamente utilizado quando são verificados problemas na multiplicação por sementes, quando é necessário fixar um determinado genótipo e evitar cruzamentos indesejados, ou ainda se deseja acelerar o processo por motivos comerciais.

Para que ocorra a indução do sistema radicial, o principal hormônio vegetal envolvido é a auxina, que age em conjunto com carboidratos, compostos nitrogenados e vitaminas. O enraizamento pode ser aumentado pela adição de auxinas sintéticas, sendo o regulador vegetal de maior uso o ácido indolbutírico (AIB) (JANICK, 1966).

A melhor época do ano em que se deve realizar a coleta do material vegetativo varia conforme o perfil de cada espécie. É claro o efeito de cada estação sobre o enraizamento das estacas, parecendo estar relacionado ao nível endógeno de auxina e que mesmo com aplicação de reguladores vegetais nas estacas, essa relação é mantida (ZUFFELLATO-RIBAS, 2001). Assim, o efeito dos reguladores vegetais aplicados pode variar conforme a estação do ano, estimulando em uma ou até inibindo em outra (IRITANI et al., 1986).

A propagação vegetativa de Kudzu, mesmo sendo uma planta com muitas finalidades, é pouco estudada. Desta forma, o objetivo deste trabalho foi estudar os efeitos da aplicação de diferentes concentrações do ácido indolbutírico (AIB) no enraizamento de estacas caulinares de Pueraria lobata (Willd) Ohwi coletadas em diferentes estações do ano, visando à obtenção de um protocolo de propagação desta espécie.

\section{MATERIAL E MÉTODOS}

O experimento foi conduzido durante o ano de 2003 na Embrapa Florestas, localizada no município de Colombo - PR. As estacas de Pueraria lobata foram coletadas no município de Piraquara, região metropolitana de Curitiba - PR, nas quatro estações do ano, ou seja, verão (10/03/03); outono (29/04/2003); inverno (22/07/2003); primavera (10/ $10 / 2003)$. As estacas foram confeccionadas com cerca de $12 \mathrm{~cm}$ de comprimento, $0,7 \mathrm{~cm}$ de diâmetro, sem folhas, com uma gema na extremidade apical, e corte em bisel na base.

Como tratamento fitossanitário prévio, as estacas foram imersas em solução de hipoclorito de sódio, a $0,5 \%$, por 5 minutos, sendo posteriormente lavadas em água corrente. Em seguida receberam tratamento com benomyl $\left(0,5 \mathrm{~g} \mathrm{~L}^{-1}\right)$, durante 15 minutos.

Suas bases foram imersas por 10 segundos em solução alcoólica (50\%), contendo diferentes concentrações de ácido indolbutírico (AIB), conforme os tratamentos $(\mathrm{T}): \mathrm{T}_{1}\left(0 \mathrm{mg} \mathrm{L}^{-1} \mathrm{AIB}\right), \mathrm{T}_{2}\left(500 \mathrm{mg} \mathrm{L}^{-1}\right.$ AIB), $T_{3}$ (1000 mg L-1 AIB), $T_{4}$ (1500 mg L-1 AIB). O plantio foi realizado em tubetes de polipropileno (53 $\mathrm{cm}^{3}$ ) com vermiculita de granulometria média como substrato, mantidos em casa-de-vegetação com sistema de nebulização intermitente num regime de 5 segundos por 10 minutos de intervalo.

O delineamento utilizado foi o inteiramente casualizado com 4 tratamentos, sendo cada tratamento constituído de 4 repetições de 24 estacas, 
num total de 384 estacas por estação. As avaliações foram realizadas 30 dias após a instalação de cada experimento, considerando as seguintes variáveis: porcentagem de estacas enraizadas; número médio de raízes por estaca; comprimento das 4 maiores raízes por estaca; porcentagem de estacas com calos (sem raízes e com calos); porcentagem de estacas vivas (sem raízes ou calos) e porcentagem de estacas mortas. Os resultados foram submetidos à analise de variância pelo teste $\mathrm{F}$ e as médias foram comparadas pelo teste de Tukey, ao nível de $5 \%$ de probabilidade.

\section{RESULTADOS E DISCUSSÃO}

Nos resultados referentes ao experimento do verão/2003, realizado no final do período de crescimento vegetativo da planta, foi possível observar que os tratamentos $\mathrm{T}_{3}\left(1000 \mathrm{mg} \mathrm{L}^{-1} \mathrm{AIB}\right) \mathrm{e}$ $\mathrm{T}_{4}$ (1500 mg L-1 AIB), mostraram-se mais eficientes quanto à porcentagem de enraizamento das estacas de Pueraria lobata (WILLD) Ohwi, quando comparados com o tratamento testemunha, resultando em 61,46 e $73,96 \%$ respectivamente, enquanto no $T_{1}$, somente $14,58 \%$ das estacas enraizaram. O mesmo ocorreu com o número médio de raízes por estaca, em que para os tratamentos $T_{3}$ e $\mathrm{T}_{4}$ foram registrado os valores 14,04 e 14,14 respectivamente, e 3,53 para $\mathrm{T}_{1}$ (Tabela 1).

Com o aumento da quantidade de raízes por estaca, possivelmente ocorreu maior absorção de água, evitando sua desidratação e ocasionando maior sobrevivência das mesmas, uma vez que houve diminuição significativa da mortalidade destas nas maiores concentrações de AIB. Quanto ao comprimento médio das quatro maiores raízes por estaca, não foi possível observar aumentos significativos entre os tratamentos indicando que 0 AIB não foi efetivo sobre esta variável (Tabela 1). LIU et al., 2002, relatam que em P. lobata, a formação de raízes laterais é altamente estimulada pela aplicação de auxinas exógenas, particularmente com AIA e AIB, mas a elongação das raízes varia drasticamente com a concentração usada no tratamento, chegando a apresentar tendência à inibição.

A baixa porcentagem de estacas vivas (sem calos e sem raízes) e de estacas com calos (sem raízes e com calos), não permitiu a análise estatística que, possivelmente, resultaria em diferenças não estatísticas. Possivelmente a baixa porcentagem de estacas vivas, pode ter se dado, em parte pela intensa diferenciação celular na formação de tecidos radiciais, em decorrência do maior acúmulo de cofatores do enraizamento presentes no período de crescimento vegetativo da planta. São considerados co-fatores do enraizamento substâncias endógenas capazes de atuar sinergisticamente com as auxinas atuando como promotores ou inibidores deste processo (HARTMANN et al., 2002). Já a baixa porcentagem de estacas com calos provavelmente se deve ao fato da rizogênese nesta espécie ser rápida e direta, quando a formação de raízes adventícias ocorre pela diferenciação de células próximas ao sistema vascular e não pela diferenciação das células dos calos (ALVARENGA; CARVALHO, 1983; HARTMANN et al., 2002).

No outono/2003, as maiores porcentagens de enraizamento foram verificadas em $\mathrm{T}_{2}(500 \mathrm{mg} \mathrm{L}$ ${ }^{1}$ AIB) e $T_{3}\left(1000 \mathrm{mg} \mathrm{L}^{-1} \mathrm{AIB}\right)$, com 53,76 e $59,79 \%$ de estacas enraizadas, respectivamente, superando os $17,30 \%$ do $T_{1}$. O número médio de raízes por estaca aumentou significativamente para as maiores concentrações, com 7,97 ( $\left.T_{3}-1000 \mathrm{mg} \mathrm{L}^{-1} \mathrm{AIB}\right)$ e 8,24 ( $T_{4}-1500 \mathrm{mg} \mathrm{L}^{-1}$ AIB) raízes por estaca, os quais diferiram de $T_{1}$ e $T_{2}$ Já para o comprimento médio das raízes, $T_{2}$ diferiu estatisticamente dos demais tratamentos, com 1,85 cm (Tabela 1).

Quando analisada a porcentagem de estacas vivas (sem raízes e sem calos), a testemunha $\left(T_{1}\right)$ mostrou diferença significativa entre os demais tratamentos, apresentando a maior porcentagem $(64,17 \%)$, demonstrando assim a ação do regulador vegetal utilizado na indução radicial, já que para os demais tratamentos foi maior a porcentagem de estacas enraizadas, reduzindo, portanto a porcentagem de estacas vivas. Não houve diferença estatística para a porcentagem de estacas com calos e mortas (Tabela 1).

No inverno/2003, ocorreram em todos os tratamentos altas porcentagens de mortalidade das estacas $(79,17 \%$ a $83,86 \%)$, provavelmente devido à suscetibilidade destas frente às baixas temperaturas, quando as matrizes de Kudzu entram em dormência (ROCHA et al., 1964; NEME, 1966; HUGHES et al., 1978), diminuindo seu metabolismo e interferindo na translocação de fotoassimilados e dos próprios reguladores vegetais, dificultando seu enraizamento (Tabela 1). As porcentagens de enraizamento e número de raízes por estaca, praticamente nulos, permitem verificar o insignificante crescimento vegetativo, próprio do período hibernal, demonstrado também pela baixa taxa de sobrevivência das estacas, a qual não diferiu estatisticamente entre os tratamentos (Tabela 1).

$\mathrm{Na}$ primavera/2003, as maiores porcentagens de enraizamento foram verificadas nos tratamentos $\mathrm{T}_{3}$ e $\mathrm{T}_{4}\left(1000 \mathrm{mg} \mathrm{L}^{-1}\right.$ AIB e $1500 \mathrm{mg} \mathrm{L}^{-1}$ AIB), com 48,62 e $64,06 \%$ de estacas enraizadas, respectivamente, diferindo estatisticamente da testemunha $\left(T_{1}\right)$ com 11,46 \%. O maior número de raízes por estaca também foi encontrada nas maiores concentrações de AIB, atingindo a média de 12,13 raízes por estaca no tratamento $T_{4}$, significativamente superior a $\mathrm{T}_{1}$ e $\mathrm{T}_{2}\left(500 \mathrm{mg} \mathrm{L}^{-1} \mathrm{AIB}\right)$ (Tabela 1$)$.

Nesta estação, a porcentagem de estacas vivas (sem raízes e sem calos), foi maior para $T_{1}$ $(17,71 \%)$, do que para os demais tratamentos. Com relação às estacas com calos, houve diferença estatística entre os tratamentos $\mathrm{T}_{2}(17,71 \%)$ e $\mathrm{T}_{4}$ (2,08\%) (Tabela 1).

A porcentagem de estacas mortas da testemunha $\left(T_{1}\right)$ atingiu $57,29 \%$ de mortalidade, e foi significativamente superior do que de $\mathrm{T}_{2}(37,50 \%)$ e $\mathrm{T}_{4}(31,77 \%)$. Existe a possibilidade que a menor 
BORTOLINI, M. F. et al. Enraizamento de Estacas...

\begin{abstract}
TABELA 01 - Resultados obtidos para médias das porcentagens de estacas de Pueraria lobata (Willd) Ohwi enraizadas, número de raízes por estaca, comprimento das quatro maiores raízes por estaca, porcentagem de estacas vivas (sem raízes e sem calos), porcentagem de estacas com calos (sem raízes e com calos) e porcentagem de estacas mortas, coletadas nas quatro estações do ano.
\end{abstract}

\begin{tabular}{|c|c|c|c|c|c|c|}
\hline \multirow[b]{2}{*}{ Tratamentos } & \multicolumn{5}{|c|}{ Estacas vivas } & \multirow[b]{2}{*}{$\begin{array}{c}\text { Estacas } \\
\text { mortas } \\
(\%)\end{array}$} \\
\hline & $\begin{array}{c}\text { Enraizadas } \\
(\%)\end{array}$ & NRE & $\begin{array}{l}\text { CRE } \\
(\mathrm{cm})\end{array}$ & $\begin{array}{c}\text { sem raízes } \\
\text { e sem calos } \\
(\%)\end{array}$ & $\begin{array}{l}\text { sem raízes e } \\
\text { com calos (\%) }\end{array}$ & \\
\hline \multicolumn{7}{|l|}{ Verão/2003 } \\
\hline $\mathrm{T}_{1} 0 \mathrm{mg} \mathrm{L}^{-1}$ & $14,58 \mathrm{c}$ & $3,53 \mathrm{~b}$ & $5,22 \mathrm{a}$ & 1,04 & 0,00 & $84,38 \mathrm{a}$ \\
\hline $\mathrm{T}_{2} 500 \mathrm{mg} \mathrm{L}^{-1}$ & 29,49 bc & $10,68 a b$ & $4,69 \mathrm{a}$ & 0,00 & 0,00 & $66,67 a b$ \\
\hline $\mathrm{T}_{3} 1000 \mathrm{mg} \mathrm{L}^{-1}$ & $61,46 a b$ & $14,04 \mathrm{a}$ & $4,45 \mathrm{a}$ & 1,04 & 1,04 & $36,46 \mathrm{bc}$ \\
\hline $\mathrm{T}_{4} 1500 \mathrm{mg} \mathrm{L}^{-1}$ & $73,96 \mathrm{a}$ & $14,14 \mathrm{a}$ & $4,21 \mathrm{a}$ & 0,00 & 1,04 & $25,00 \mathrm{c}$ \\
\hline CV(\%) & 38,79 & 33,49 & 26,38 & - & - & 31,98 \\
\hline \multicolumn{7}{|l|}{ Outono/2003 } \\
\hline $\mathrm{T}_{1} 0 \mathrm{mg} \mathrm{L}^{-1}$ & $17,30 \mathrm{~b}$ & $2,20 \mathrm{c}$ & $1,01 \mathrm{~b}$ & $64,17 \mathrm{a}$ & $10,01 \mathrm{a}$ & $8,52 a^{*}$ \\
\hline $\mathrm{T}_{2} 500 \mathrm{mg} \mathrm{L}^{-1}$ & $53,76 a$ & $5,05 \mathrm{~b}$ & $1,85 \mathrm{a}$ & $15,21 b$ & $17,30 \mathrm{a}$ & $13,71 \mathrm{a}$ \\
\hline $\mathrm{T}_{3} 1000 \mathrm{mg} \mathrm{L}^{-1}$ & $59,79 a$ & $7,97 \mathrm{a}$ & $1,32 b$ & $13,96 b$ & $15,00 \mathrm{a}$ & $11,28 \mathrm{a}$ \\
\hline $\mathrm{T}_{4} 1500 \mathrm{mg} \mathrm{L}^{-1}$ & $38,08 \mathrm{ab}$ & $8,24 \mathrm{a}$ & $1,14 \mathrm{~b}$ & $19,33 \mathrm{~b}$ & 26,62 a & $15,97 \mathrm{a}$ \\
\hline $\mathrm{CV}(\%)$ & 23,43 & 20,26 & 18,48 & 29,08 & 47,20 & 67,40 \\
\hline \multicolumn{7}{|l|}{ Inverno/2003 } \\
\hline $\mathrm{T}_{1} 0 \mathrm{mg} \mathrm{L}^{-1}$ & 0,00 & 0,00 & 0,00 & $14,06 \mathrm{a}$ & 2,08 & $83,86 \mathrm{a}$ \\
\hline $\mathrm{T}_{2} 500 \mathrm{mg} \mathrm{L}^{-1}$ & 0,00 & 0,00 & 0,00 & $17,71 \mathrm{a}$ & 0,00 & $82,29 a$ \\
\hline $\mathrm{T}_{3} 1000 \mathrm{mg} \mathrm{L}^{-1}$ & 1,04 & 1,00 & 0,36 & $17,71 \mathrm{a}$ & 2,08 & $79,17 \mathrm{a}$ \\
\hline $\mathrm{T}_{4} 1500 \mathrm{mg} \mathrm{L}^{-1}$ & 1,04 & 0,25 & 0,38 & $19,06 \mathrm{a}$ & 0,00 & $79,90 \mathrm{a}$ \\
\hline $\mathrm{CV}(\%)$ & - & - & - & 40,11 & - & 15,02 \\
\hline \multicolumn{7}{|l|}{ Primavera/2003 } \\
\hline $\mathrm{T}_{1} 0 \mathrm{mg} \mathrm{L}^{-1}$ & $11,46 \mathrm{c}$ & $1,52 \mathrm{c}$ & $3,29 a$ & $17,71 \mathrm{a}$ & $13,54 a b$ & $57,29 a$ \\
\hline $\mathrm{T}_{2} 500 \mathrm{mg} \mathrm{L}^{-1}$ & $39,58 \mathrm{~b}$ & $3,23 \mathrm{bc}$ & $1,63 \mathrm{a}$ & $5,21 \mathrm{~b}$ & $17,71 \mathrm{a}$ & $37,50 \mathrm{~b}$ \\
\hline $\mathrm{T}_{3} 1000 \mathrm{mg} \mathrm{L}^{-1}$ & $48,62 \mathrm{ab}$ & $7,68 a b$ & $3,01 \mathrm{a}$ & $1,04 \mathrm{~b}$ & $4,86 a b$ & $45,48 a b$ \\
\hline $\mathrm{T}_{4} 1500 \mathrm{mg} \mathrm{L}^{-1}$ & $64,06 \mathrm{a}$ & $12,13 \mathrm{a}$ & $2,97 \mathrm{a}$ & $2,08 \mathrm{~b}$ & $2,08 \mathrm{~b}$ & $31,77 b$ \\
\hline $\mathrm{CV}(\%)$ & 18,43 & 36,35 & 32,89 & 30,46 & 35,08 & 20,14 \\
\hline
\end{tabular}

Médias seguidas pela mesma letra na coluna não diferem estatisticamente para teste de Tukey ao nível de $5 \%$ de probabilidade.

NRE: Número médio de raízes por estaca; CRE: comprimento médio das quatro maiores raízes por estaca (cm). * Médias transformadas para arc seno $x^{1 / 2}$. 
mortalidade verificada na primavera com relação ao experimento do inverno, se deva à maior presença de fotoassimilados acumulados e a temperaturas ambientais mais amenas, possibilitando um maior aproveitamento nutricional e hídrico pela planta matriz (VEIERSKOV, 1988; HAMBRICK et al., 1991; PALANISAMY e SUBRAMANIAN, 2001). Quando analisada a variável comprimento médio das quatro maiores raízes por estaca na primavera, não houve diferença significativa entre os tratamentos (Tabela $1)$.

A influência da estação do ano sobre a indução radicial, provavelmente foi causado devido à diferença nas estacas quanto às reservas de nutrientes nos tecidos cambiais e da atividade cambial, e distribuição de auxinas endógenas na estaca (PURUSHOTHAM et al., 1984; PAL, 1990). Assim, o efeito das auxinas exógenas pode variar nas diferentes épocas do ano, agindo ora como estimulante, ora como inibidor, podendo tornar-se fitotóxico em alguns casos (ZUFFELLATO-RIBAS, 2001), já que a habilidade ao enraizamento de uma estaca caulinar é determinada pelo balanço entre fatores nutricionais e hormonais e que o enraizamento pode vir a não ocorrer quando a concentração de um destes estiver muito alta (NANDA et al., 1971).

No entanto, Doran (1947), estudando a estaquia de kudzu, conseguiu $100 \%$ de enraizamento com estacas coletadas no outono e tratadas com Hormodin $\mathrm{n}-1$, produto comercial que contem $1000 \mathrm{mg} \mathrm{kg}^{-1}$ de ácido indolbutirico (AIB) sob a forma de talco; para as estacas coletadas no verão obteve $67 \%$ de enraizamento, mas quando na mesma estação tratou as estacas com Hormodin no II, que contem $3000 \mathrm{mg} \mathrm{kg}^{-1}$ de AIB, obteve $87 \%$ de enraizamento. No inverno foi registrada grande mortalidade das estacas, comprovando o resultado obtido no presente trabalho.
A diferença entre os resultados obtidos por DORAN (1947), com os do presente trabalho, poderia ser justificada pela diferença na forma de aplicação do AIB, se em talco ou solução. Geralmente têm se observado que a resposta à forma de aplicação dos reguladores vegetais é variável para as espécies.

\section{CONCLUSÃO}

Nas condições em que foi desenvolvido o presente trabalho, foi possível concluir que o uso de ácido indolbutírico (AIB) estimula o enraizamento de estacas caulinares de Pueraria lobata (Willd) Owhi, recomendando-se a aplicação de $1000 \mathrm{mg} \mathrm{L}^{-1} \mathrm{AlB}$, em qualquer estação do ano, com exceção do inverno.

\section{AGRADECIMENTOS}

Os autores consignam sinceros agradecimentos aos Srs. Vero Oscar Cardoso dos Santos, Carlos Amílcar de Carvalho Silva e Paulino Graff, da Embrapa Florestas, pela ajuda constante na coleta de material e instalação dos experimentos.

\section{REFERÊNCIAS}

1. ALVARENGA, L.R.; CARVALHO, V.D. Uso de substâncias promotoras de enraizamento de estacas frutíferas. Informe Agropecuário, Belo Horizonte, v. 9, n. 101, p. 47-55, 1983.

2. APIRS, Center for Aquatic and Invasive Plants. Pueraria montana (Lour.) Merr. var. Iobata (Willd.) Maesen \& S. M. Almeida . University of Florida, IFAS. Disponível em: <http://plants.ifas.ufl.edu/puemon.pdf>. Acesso em 12 jan. 2003.

3. CORRÊA, P.M. Dicionário das plantas úteis do Brasil e das exóticas cultivadas . v. 4. Rio de Janeiro: IBDF, 1969.607 p.

4. DCR, Department of Conservation and Recreation, Richmond, Virginia. Invasive alien plant species of Virginia. Disponível em: <http://www.dcr.state.va.us/dnh/fspulo.pdf>. Acesso em 26 mar. 2003.

5. DORAN, W.L. Vegetative propagation of kudzu. Journal of the American Society of Agronomy, Washington, v. 39, n. 9, p. 834-835, 1947.

6. FORSETH, I.N; INNIS, A.F. Kudzu (Pueraria Montana): history, physiology, and ecology combince to make a major ecosystem threat. Critical Reviews in Plant Sciences, Philadelphia, v. 23, n. 5, p. 401-413, 2004.

7. HAMBRICK, C.E.; DAVIES, F.T.; PEMBERTON, H.B. Seasonal changes in carbohydrate/nitrogen levels during field rooting of Rosa multiflora "Brooks 56" hardwood cuttings. Scientia Horticulturae, Amsterdam, v. 46, p. 137-146, 1991.

8. HARTMANN, H.T.; KESTER, D.E.; DAVIS JÚNIOR, F.T; GENEVE, R.L. Plant propagation: principles and practices. 7 ed. New York: Englewood Clipps, 2002. 880 p.

9. HUA-NENG, $\mathrm{X}$; CHAO-HONG, H. Extraction of isoflavones from stem of Pueraria lobata (Willd.) Ohwi using n-butanos/water two-phase solvent system and separation of daidzein. Separation and Purification Technology, Amsterdam, v. 56, p. 81-85, 2007.

10. HUGHES, H.D.; HEATH, M.E.; METIALFE, D.J. Forrajes: the science of grassland agriculture. 8. ed. México: Compania Editorial Continental J.A., 1978. 247 p.

11. IRITANI, C.; SOARES, R.V.; GOMES, A. V. Aspectos morfológicos da aplicação de reguladores do crescimento nas estacas de Ilexparaguariensis St. Hilaire. Acta Biológica Paranaense, Curitiba, v. 15, p. 21-46, 1986.

12. JANICK, J. A ciência da horticultura. Rio de Janeiro: F. Bastos, 1966. $485 p$ 
BORTOLINI, M. F. et al. Enraizamento de Estacas...

13. (K.K.D.S, INC): KUDZU KINGDOW DIVISION OF SUNTOP, INC. Kudzu products and publications . Disponível em: <http:/ / www.kudzukingdom.com>. Acesso 20 nov. 2002.

14. LIU, C.; ZHU, J.; LIU, Z.;LI, L.; PAN, R.; JIN, L. Exogenous auxin effects on growth and phenotype of normal and hairy roots of Pueraria lobata (Willd.) Ohwi. Plant Growth Regulation, Dordrecht, n. 38, p. 37-43, 2002.

15. MUNGER, G.T. Pueraria montana var. Lobata. U.S. Department of Agriculture, Forest Service, Rocky Mountain Research Station, Fire Sciences Laboratory. Disponível em:<http://www.fs.fed.us/database/feis/plants/vine/puemonl/index.html>. Acesso em 28 mar.2003.

16. NANDA, K.K.; JAIN, M.K.; MALHOTRA, S. Effect of glucose and auxins in rooting etiolated stem segments of Populus nigra. Physiology Plantarum, Copenhagem, v. 24, p. 387-391, 1971.

17. NEME, A.N. Leguminosas para adubos verdes e forragens . 4. ed, Campinas: IAC, 1966. p. 18-20. (Boletim 109).

18. PAEZ, J.C. Algunas consideraciones sobre el kudzu. Estación Experimental de La Molina: Ministerio de Agricultura Perú, 1946. 34 p. (Circular, 63).

19. PAL, M. Seasonal variations of the effects of auxins on rooting branch cuttings of Hibiscus rosasiensis. Indian Journal of Forestry, Dehra Dun, v. 13, n. 4, p. 333-335, 1990.

20. PALANIASAMY, K.; SUBRAMANIAN, K. Vegetative propagation of mature teak trees ( Tectona grandis L.). Silvae Genética, Frankfurt am Main, v. 50, n. 5, p. 188-191, 2001.

21. PURUSHOTHAM, K.; SULLADMATH, U.V.; RAMAIAH, P.K. Seasonal changes in biochemical constituents and their relation to rooting of Coffe (Coffe canephora Pierre) sucker cuttings. Journal of Coffe Reserch, Mysore, v. 14, n. 3, p. 117-130, 1984.

22. ROCHA, G.L. Cudzú: fForrageira para solos pobres e de mediana fertilidade. Série de Vulgarização, Plantas Forrageiras, São Paulo, v. 6, n.10, p. 54- 70, 1964.

23. ROMM, H.J. The development and structure of the vegetative and reprodutive organs of kudzu, Pueraria thumbergiana(Sieb. and Zucc.) Benth. Bulletin lowa Agricultural Experiment Station, Ames, v. 27, n. 3, p. 407-419, 1953.

24. STARR F.; MARTZ K.; LOOPE L. Kudzu ( Pueraria lobata): an alien plant report. United States Geological Survey Biological Resources Division. Disponível em:<http://www.hear.org/species/reports/puelob_fskm_awwa_report.pdf>. Acesso em 27 mar. 2003.

25. THIEM, B. In vitro propagation of isoflavone-producing Pueraria lobata (Willd.) Ohwi. Plant Science, Lincoln, v. 106, p. 1123$1128,2003$.

26. VEIERSKOV, B. Relations between carbohydrates and adventitious root formation. In: DAVIS, T.D.; HAISSIG, B.E.; SANKHLA N. Adventitious root formation in cuttings. v. 2. Oregon: Dioscorides Press, 1988. $315 \mathrm{p}$.

27. ZUFFELLATO-RIBAS, K.C.; RODRIGUES, J.D. Estaquia: uma abordagem dos principais aspectos fisiológicos. Curitiba: K.C. Zuffellato-Ribas?, 2001.39p.

Recebido em 17/11/2006

Aceito em 15/05/2007 